\title{
High Oxygen Nanocomposite Barrier Films Based on Xylan and Nanocrystalline Cellulose
}

\author{
Amit Saxena ${ }^{1}$, Thomas J. Elder ${ }^{2}$, Jeffrey Kenvin ${ }^{3}$ and Arthur J. Ragauskas ${ }^{1, *}$
}

The goal of this work is to produce nanocomposite film with low oxygen permeability by casting an aqueous solution containing xylan, sorbitol and nanocrystalline cellulose. The morphology of the resulting nanocomposite films was examined by scanning electron microscopy and atomic force microscopy which showed that control films containing xylan and sorbitol had a more open structure as compared to xylan-sorbitol films containing sulfonated nanocrystalline cellulose. The average pore diameter, bulk density, porosity and tortuosity factor measurements of control xylan films and nanocomposite xylan films were examined by mercury intrusion porosimetry techniques. Xylan films reinforced with nanocrystalline cellulose were denser and exhibited higher tortuosity factor than the control xylan films. Control xylan films had average pore diameter, bulk density, porosity and tortuosity factor of $0.1730 \mu \mathrm{m}, 0.6165 \mathrm{~g} / \mathrm{ml}, 53.0161 \%$ and 1.258 , respectively as compared to xylan films reinforced with $50 \%$ nanocrystalline cellulose with average pore diameter of $0.0581 \boldsymbol{\mu m}$, bulk density of $1.1513 \mathrm{~g} / \mathrm{ml}$, porosity of $22.8906 \%$ and tortuosity factor of 2.005 . Oxygen transmission rate tests demonstrated that films prepared with xylan, sorbitol and 5\%, 10\%, 25\% and $50 \%$ sulfonated nanocrystalline cellulose exhibited a significantly reduced oxygen permeability of $1.1387,1.0933,0.8986$ and $0.1799 \mathrm{~cm}^{3} \cdot \mu \mathrm{m} / \mathrm{m}^{2} \cdot \mathrm{d} \cdot \mathrm{kPa}$ respectively with respect to films prepared solely from xylan and sorbitol with a oxygen permeability of $189.1665 \mathrm{~cm}^{3} \cdot \mu \mathrm{m} / \mathrm{m}^{2} \cdot \mathrm{d} \cdot \mathrm{kPa}$. These properties suggested these nanocomposite films have promising barrier properties.

Keywords: Nanocomposites; Xylan; Nanocrystalline cellulose; Oxygen barrier

Citation: Amit Saxena, Thomas J. Elder, Jeffrey Kenvin and Arthur J. Ragauskas, "High Oxygen Nanocomposite Barrier Films Based on Xylan and Nanocrystalline Cellulose", Nano-Micro Lett. 2, 235-241 (2010). doi:10.3786/nml.v2i4. p235-241

Biobased plastics have generated increasing interest to replace petrochemical based polymers in light of their ready availability, sustainability, limited environmental impact and simplified end-of-life disposal issues [1-4]. High barrier packaging materials require low oxygen permeability with good mechanical strength. Replacing existing oxygen barriers such as aluminum and synthetic polymers with biopolymers is a research topic of increased interest. Early studies examined the use of chitosan, starch and cellulose derivatives to address these challenges and were shown to have good film forming properties [5].

Acetylated galactoglucomannan (AcGGM) hemicellulose was found to be an excellent candidate for making new renewable barrier materials [6]. The oxygen barrier permeability of the AcGGM films were found to be similar to, or lower than, the values reported on oxygen barrier films made from glucuronoxylan [7] and other polysaccharides, such as starch [8], chitosan [9] and mixtures of various polysaccharides [10]. Hartman reported oxygen permeability of $2.0 \mathrm{~cm}^{3} \cdot \mu \mathrm{m} / \mathrm{m}^{2} \cdot \mathrm{d} \cdot \mathrm{kPa}$ for GGM-sorbitol film [6]. The oxygen barrier properties of films obtained from a mixture of $O$-acetyl-galactoglucomannan and either alginate or carboxymethylcellulose were also studied. The reported values of oxygen permeability of GGM/alginate/ 
glycerol blend were $4.6 \mathrm{~cm}^{3} \cdot \mu \mathrm{m} / \mathrm{m}^{2} \cdot \mathrm{d} \cdot \mathrm{kPa}$ [6]. The oxygen permeability of the GGM films was lower than that of glycerol-plasticized amylose and amylopectin films [11], but not as low as that of sorbitol-plasticized aspen glucuronoxylan films $[7,12]$. The oxygen permeability of oat spelt arabinoxylan films plasticized with $40 \%$ sorbitol was $4.7 \mathrm{~cm}^{3} \cdot \mu \mathrm{m} / \mathrm{m}^{2} \cdot \mathrm{d} \cdot \mathrm{kPa}$, which is slightly lower than that of GGM films [13]. Biobased free-standing films and coatings with low oxygen permeability of $1 \mathrm{~cm}^{3} \cdot \mu \mathrm{m} / \mathrm{m}^{2} \cdot \mathrm{d} \cdot \mathrm{kPa}$ have been also prepared from a wood hydrolysate [14]. Films made from these polysaccharides are brittle and therefore to form cohesive films requires plasticizers such as sorbitol and xylitol. However, even with the plasticizers, the mechanical properties of these films have been considered to less than desirable. Nanocrystalline cellulose has been studied as reinforcements of various synthetic and some natural polymer matrices [15-17] to improve the strength properties owing to their high bending strength of $10 \mathrm{GPa}$, and elastic modulus of $143 \mathrm{GPa}$ [18-19]. Lagaron et al. [20] discussed the role of crystalline structure of polymers and emphasized that high crystallinity improves barrier properties. Nanocrystalline cellulose is greater than $60 \%$ crystalline [21-22] and this property together with the resulting rigid hydrogen-bonded network of nanocrystallinecellulose can cause an increase in tortuousity and smaller pore size for nanocomposites which may be utilized to create high barrier materials. In a recent study, spruce galactoglucomannans (GGM) and konjac glucomannan (KGM) were mixed with nanocrystalline cellulose (NCC) to study the mechanical and barrier properties of the films [23,24]. The tensile strength of unplasticized KGM films increased by $30 \%$ but the mechanical properties of the plasticized films were not affected with increased in nanocrystalline cellulose. The presence of $5 \%$ of nanocrystalline cellulose did not significantly affect the oxygen permeability of the films.

Xylan is one of the most common hemicelluloses, is the most abundant polysaccharide in nature after cellulose, and is an attractive resource for film production [25-27]. Prior studies by Saxena et al. [28] have shown that the addition of $7 \%$ nanocrystalline cellulose to xylan-sorbital films increased the tensile energy absorption of the resulting films by $445 \%$ and the tensile strength of the film by $141 \%$. Recent studies demonstrated that films with $10 \%$ nanocrystalline cellulose exhibited a $74 \%$ reduction in specific water transmission properties with respect to films prepared solely from xylan/sorbitol, and a $362 \%$ reduction in water transmission rate with respect to xylan films reinforced with $10 \%$ softwood kraft fibers [29]. The objective of the current study is to evaluate the oxygen barrier properties of xylan-nanocrystalline cellulose composite films.

\section{Materials and Methods}

\section{Materials}

Oat spelt xylan was obtained from Aldrich and was determined to contain $81.0 \%$ xylose, $9.8 \%$ arabinose, $7.6 \%$ glucose, $1.4 \%$ galactose and $0.2 \%$ of mannose. A commercial elemental chlorine-free (ECF) bleached softwood (SW) kraft pulp was used as received. Dialysis tubes were purchased from Spectrum Labs. All other reagents and solvents were purchased from Aldrich and used as received.

\section{Preparation of Nanocrystalline Cellulose}

Nanocrystalline cellulose was prepared following the procedure outlined by $\mathrm{Pu}$ [30]. In brief, softwood kraft pulp was ground in a Wiley mill to pass through a 20 -mesh screen. The acid hydrolysis of the milled pulp was accomplished with 64 wt $\%$ sulfuric acid at $45^{\circ} \mathrm{C}$ for $45 \mathrm{~min}$ with stirring. The reaction was halted by adding a 10 -fold excess of deionized (DI) water. After standing overnight, the water was decanted and the settled cellulosics were collected with a minimal amount of water. Excess acid was removed with a water wash $(200 \mathrm{ml})$, and centrifuged for $20 \mathrm{~min}$ at 11,000 rpm. The aqueous phase was discarded and the wash cycle was repeated an additional two times. The solids suspension was then dialyzed against DI water for 3 days using regenerated cellulose dialysis tubing with a 12000-14000 molecular weight cut off until the $\mathrm{pH}$ of the solution reached 7. Sonication was performed on the neutral nanocrystalline cellulose solution for $35 \mathrm{~min}$ with an Ultrasonic Processor GEX-500 sonicator with standard titanium alloy probe (tip diameter $1.27 \mathrm{~cm}$ ) at 28 Watts power input while immersed in an ice bath. The average yield of nanocrystalline cellulosic was $35 \%$. Conductometric titration was used to measure the bulk charge on nanocrystalline cellulose following the method described by Katz (1984) [31]. A known amount of nanocrystalline cellulose $(0.31 \%$ of suspension containing $0.50 \mathrm{~g}$ of whisker) was taken and mixed with $5.00 \mathrm{ml}$ of $0.01 \mathrm{M} \mathrm{NaCl}$ before measurement. The solution was titrated with $0.05 \mathrm{M}$ $\mathrm{NaOH}$.

The change in conductivity was recorded with a conductometer. The bulk charge for the nanocrystalline cellulose was found to be $9.73 \mathrm{mmol}$ acid groups $/ 100 \mathrm{~g}$. The bulk carboxylic acid group content of the fibers was measured by 
conductometric titration methodology. Air-dried fibers equivalent to $1.50 \mathrm{~g}$ of oven-dried fibers were added to 300.00 $\mathrm{ml}$ of $0.10 \mathrm{M} \mathrm{HCl}$ and stirred for $1 \mathrm{~h}$. The pulp was filtered using a vacuum flask and then washed with at least $400 \mathrm{ml}$ of deionized water. The washed pulp was treated with $0.001 \mathrm{M} \mathrm{NaCl}$ and 1.50 $\mathrm{mL}$ of $0.10 \mathrm{M} \mathrm{HCl}$, stirring and bubbling nitrogen through the solution. The treated pulp was finally titrated with $0.05 \mathrm{M} \mathrm{NaOH}$ in $0.25 \mathrm{ml}$ aliquots. The change in conductivity was recorded with conductometer. The bulk charge for the starting cellulose fibers was found to be $3.82 \mathrm{mmol}$ carboxylate groups/100 g.

\section{Preparation of xylan- sulfonated nanocrystalline cellulose} nanocomposite film

Xylan composite films were formed by adding an aqueous suspension $(35.00 \mathrm{ml})$ of sulfonated nanocrystalline cellulose to xylan $(0.25 \mathrm{~g})$. The solid nanocrystalline cellulose content was $0.0,5.0,10.0,25.0$, and $50.0 \mathrm{wt} \%$ of the total mixture of sorbitol, xylan, and nanocrystalline cellulose. Sorbitol $(0.25 \mathrm{~g}, 1.37 \mathrm{mmol})$ was then added to the mixture with stirring and this solution was heated to $95^{\circ} \mathrm{C}$ for $15 \mathrm{~min}$. The whole solution was then poured into polystyrene petri dishes ( $4.60 \mathrm{~cm}$ in radius) and allowed to dry at room temperature for three days. The thickness of resulting composite films for xylan-sulfonated nanocrystalline cellulose films with $10 \%, 25 \%$ and $50 \%$ sulfonated nanocrystalline cellulose was $0.0812 \mathrm{~mm}, 0.0877 \mathrm{~mm}, 0.1312$ $\mathrm{mm}$, respectively.

\section{Characterization}

The cross-section and surface morphology of the composite films were analyzed by Hitachi S800, thermally assisted field emission (TFE) scanning electron microscope (SEM) with an accelerating voltage of $12 \mathrm{kV}$. The samples were sputter-coated with gold prior to examination. Nanocrystalline cellulose and the composite films were analyzed using Dimension 3100 scanning probe microscope and Nanoscope III controller. The images were acquired in tapping mode in air using a 1-10 $\Omega \mathrm{cm}$ phosphorus (n) doped silicon tip with the cantilever resonance frequency of $150 \mathrm{kHz}$. Scans were done at 5 microns.

\section{Oxygen permeability Analysis}

The oxygen transmission of the films was measured using a Mocon Ox-Tran 2/21 1 apparatus (Modern Controls Inc., Minneapolis, USA) with a coulometric sensor in accordance with ASTM method D 3985-95 [32].

\section{Mercury Intrusion Porosimetry Analysis}

Micromeritics' AutoPore IV 9500 Series was used to measure the porosity, bulk density, and average pore diameter and tortuosity factor of the control and nanocomposite films.

\section{Results and Discussion}

This study examines the oxygen barrier properties of xylan-nanocrystalline cellulose composite films. By AFM analysis, the sulfonated nanocrystalline cellulosic were observed to have rod like structure with an average length of 150-200 nm and a width of less than $20 \mathrm{~nm}$ (Fig. 1). AFM images, acquired using tapping mode, of the xylan/sorbitol films reinforced with nanocrystalline cellulose show well dispersed sulfonated nanocrystalline cellulose on xylan surface in comparison to more open structure of xylan/sorbitol control films (Fig. 2).

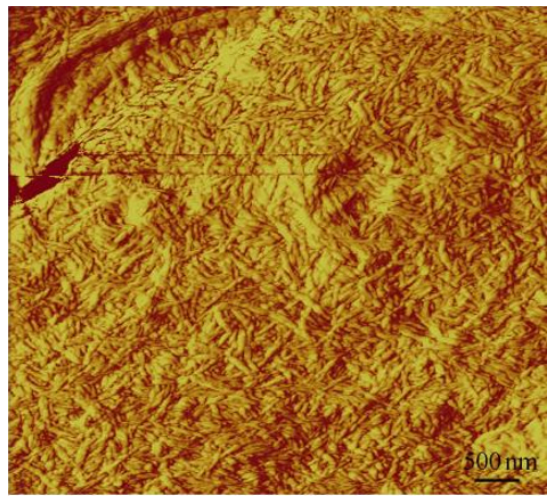

FIG. 1. AFM image of sulfonated nanocrystalline cellulose with a concentration of $0.7 \%$ by weight.

The specific oxygen transmission rate of the xylan nanocomposite films are shown in Table 1. The transmission rate decreased drastically upon reinforcement with nanocrystalline

Table 1 Effect of sulfonated nanocrystalline cellulose dosage on oxygen transmission rate of xylan film.

\begin{tabular}{lc}
\hline \multicolumn{1}{c}{ Samples } & $\begin{array}{c}\text { Specific oxygen } \\
\text { transmission rate } \\
\left(\mathrm{cm}^{3} / \mathrm{m}^{2} \cdot \text { day }\right)\end{array}$ \\
\hline Control (Xylan) & 354.950 \\
Xylan $+5 \%$ sulfonated nanocrystallinecellulose & 1.442 \\
Xylan $+10 \%$ sulfonated nanocrystalline cellulose & 1.364 \\
Xylan $+25 \%$ sulfonated nanocrystalline cellulose & 1.038 \\
Xylan $+50 \%$ sulfonated nanocrystalline cellulose & 0.139 \\
EVOH & $3-5$ \\
$\begin{array}{l}\text { Polyester, oriented, Polyvinylidene chloride (PVdC) } \\
\text { coated }\end{array}$ & $9-15$ \\
MFC film & $17.0-18.5$ \\
\hline
\end{tabular}



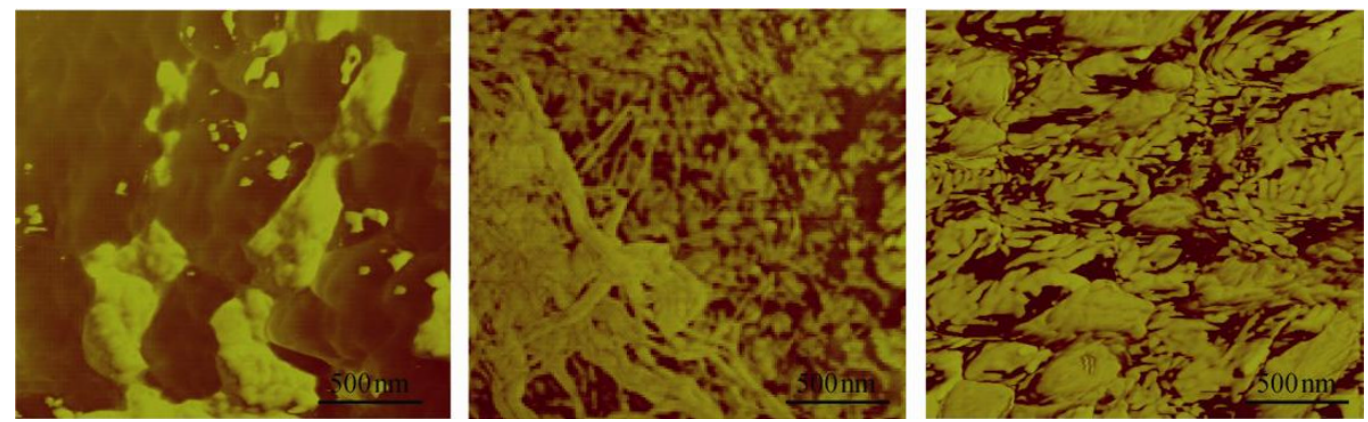

FIG. 2. AFM images of (a) control xylan (b) $10 \%$ sulfonated nanocrystalline cellulose film (c) $50 \%$ sulfonated nanocrystalline cellulose film.

cellulose. The measured oxygen permeability is lower or comparable to the often used barrier plastic ethylene vinyl alcohol (EVOH) [33] and the films made from microfibrillar cellulose [34], see Table 1.

Oxygen permeability values were calculated by dividing the oxygen transmission rates by the differential partial pressure of oxygen across the film ( 1 atm or $101.3 \mathrm{kPa}$ ) and multiplying by the film thickness in microns [5]. Table 2 summarized the oxygen permeability of some of the literature work and current work. The oxygen transmission rates as summarized in Table 1 at $25 \%$ and $50 \%$ dosage of nanocrystalline cellulose decreased drastically with respect to control xylan films and are the two lowest values that we obtained in this study. It will be an interesting subject to explore the porosity, bulk density and tortuosity factor at these two levels and the control xylan films. As summarized in Table 3, the density and tortuosity factor of the composite film increased while the pore diameter and porosity decreased as the loading of sulfonated nanocrystalline cellulose increased in the xylan-based films.

Table 2 Oxygen Permeability of biopolymer films.

\begin{tabular}{lc}
\hline \multicolumn{1}{c}{ Sample } & $\begin{array}{c}\text { Oxygen permeability } \\
\left(\mathrm{cm}^{3} \cdot \mu \mathrm{m} / \mathrm{m}^{2} \cdot \mathrm{d} \cdot \mathrm{kPa}\right)\end{array}$ \\
\hline GGM-sorbitol & $2.0[6]$ \\
GGM/alginate/glycerol blend & $4.6[6]$ \\
$\begin{array}{l}\text { Oat spelt arabinoxylan films plasticized with } 40 \% \\
\text { sorbitol }\end{array}$ & $4.7[13]$ \\
$\begin{array}{l}\text { Biobased free-standing films and coatings from a } \\
\text { wood hydrolysate }\end{array}$ & $1.0[14]$ \\
Xylan $+50 \%$ sulfonated cellulose whiskers & $0.1799($ current study $)$ \\
\hline
\end{tabular}

SEM images of the control xylan film surface showed agglomerated structures on the surface in comparison to a more uniform surface for the nanocrystalline cellulose-xylan films (Fig. 3 (a) and 3 (b)).

Oxygen transmission rate at $5 \%$ and $10 \%$ charge of nanocrystalline cellulose doesn't differ much but a significant drop of transmission rate as compared to control. We studied xylan-10\% nanocrystalline cellulose film under SEM (Fig. 3) and AFM (Fig. 2b) and found that control xylan film surface in Fig. 3 (a) shows agglomeration in comparison to well dispersed sulfonated nanocrystalline cellulose on xylan surface in Fig. 3 (b). The uneven structure and agglomeration of the xylan can be the cause of higher oxygen transmission rate of control xylan film in comparison to xylan reinforced with $10 \%$ sulfonated nanocrystalline cellulose.

Table 3. Mercury intrusion porosimetry measurements of control xylan and nano-composite films.

\begin{tabular}{ccccc}
\hline Sample & $\begin{array}{c}\text { Average pore } \\
\text { diameter }(\mu \mathrm{m})\end{array}$ & $\begin{array}{c}\text { Bulk density } \\
(\mathrm{g} / \mathrm{ml})\end{array}$ & Porosity $(\%)$ & $\begin{array}{c}\text { Tortuosity } \\
\text { factor }\end{array}$ \\
\hline $\begin{array}{c}\text { Control (Xylan) } \\
\text { Xylan+25\% } \\
\text { sulfonated }\end{array}$ & 0.1730 & 0.6165 & 53.0161 & 1.258 \\
$\begin{array}{c}\text { nanocrystalline } \\
\text { cellulose } \\
\text { Xylan+25\% } \\
\text { sulfonated } \\
\text { nanocrystalline } \\
\text { cellulose }\end{array}$ & 0.1301 & 0.8705 & 40.8501 & 1.700 \\
\hline
\end{tabular}

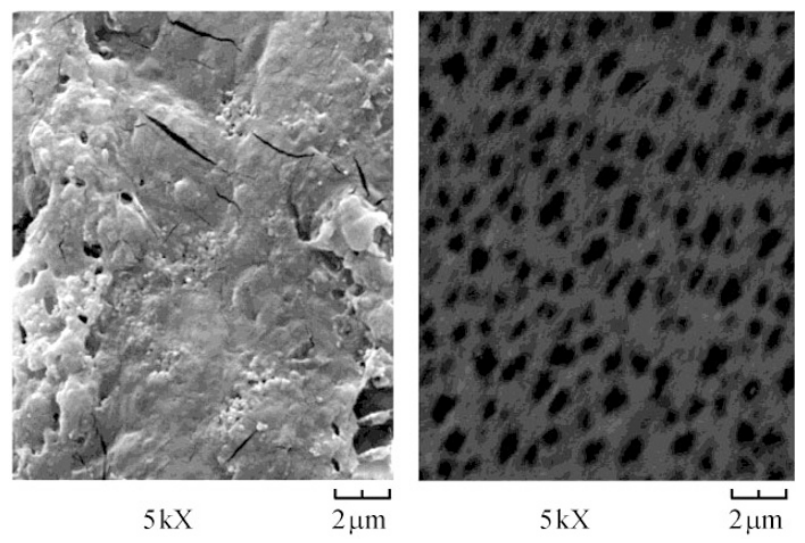

FIG. 3. SEM surface images of the (a) control xylan, (b) xylan reinforced with $10 \%$ sulfonated nanocrystalline cellulose.

SEM cross-section images of freeze fracture of control xylan films showed a rough texture with small cracks in the film as summarized in Fig. 4(a) and 4(b). The same analysis for the xylan film reinforced with nanocrystalline cellulose exhibited smooth fractured surface and less porous structure (see Fig. 4(c) and Fig. 4(d)). 


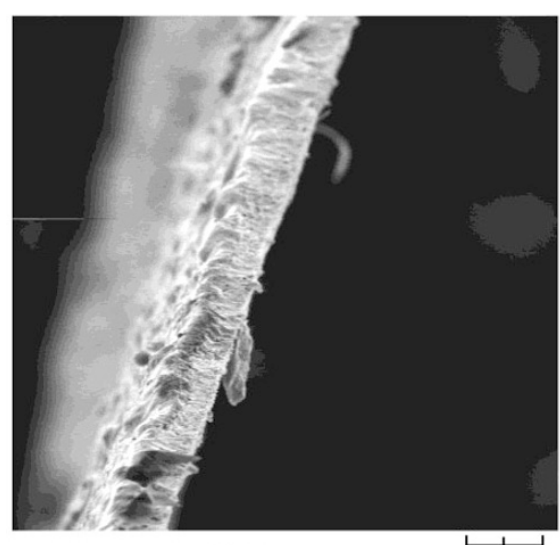

$500 \mathrm{X}$

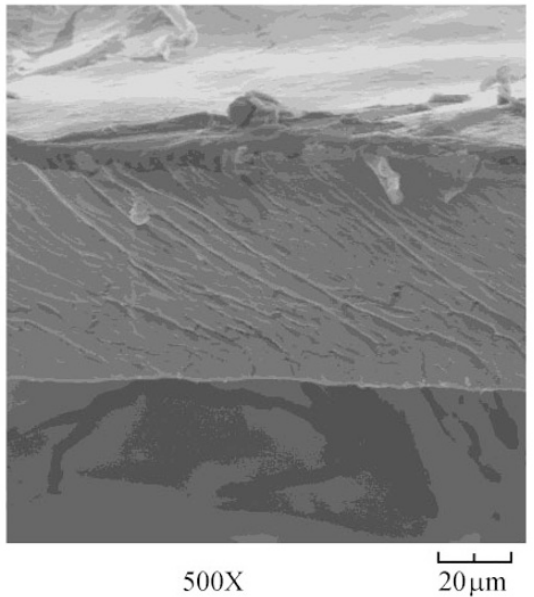

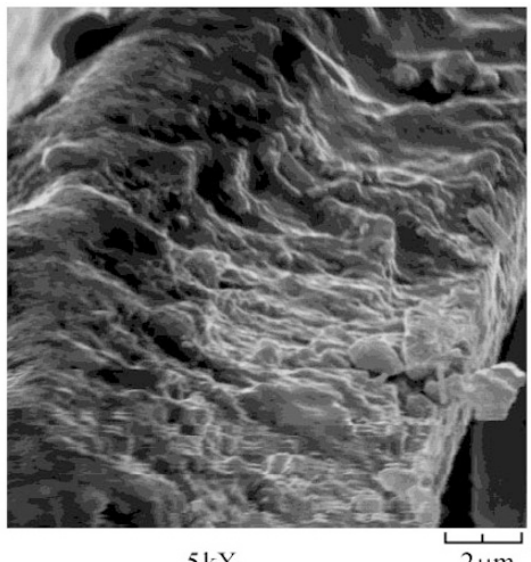

$5 \mathrm{kX}$

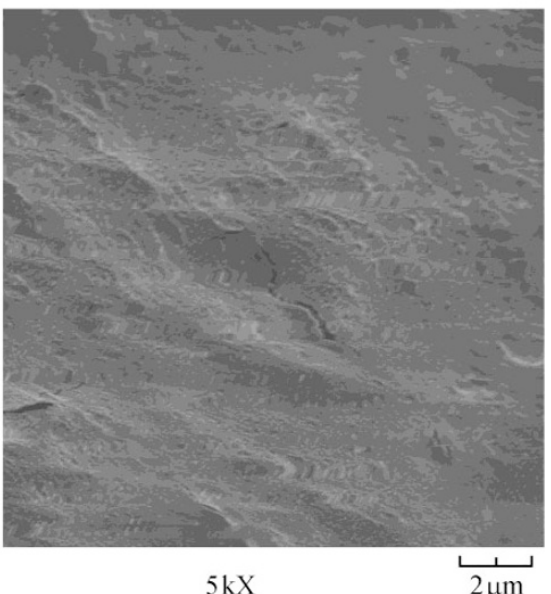

$5 \mathrm{kX}$

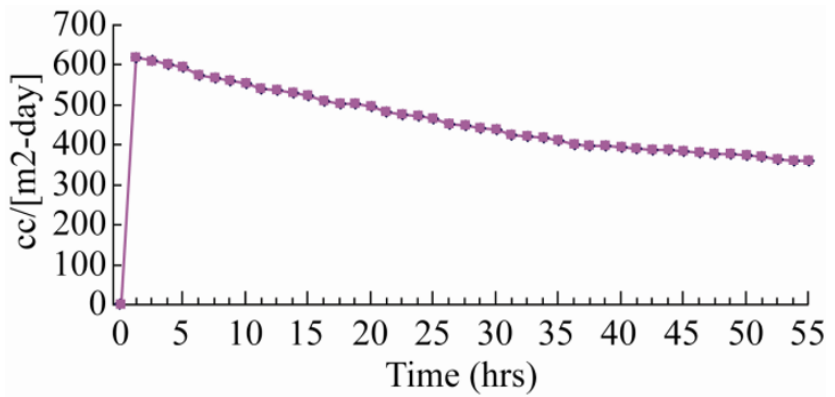

FIG. 5. Oxygen transmission rate for control xylan films.

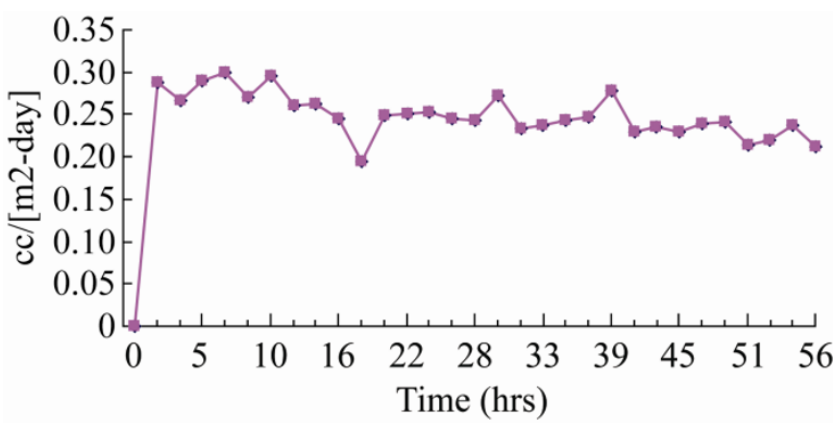

FIG. 6. Oxygen transmission rate for control xylan reinforced with $50 \%$ nanocrystalline cellulose. 


\section{Conclusions}

Nanocrystalline cellulose was incorporated into xylan slurry to prepare high oxygen barrier biodegradable films. It appears that the high degree of crystallinity of nanocrystalline cellulose and the dense composite structure of the formed films with nanocrystalline cellulose and xylan leads to a film that has reduced transmission properties. These effects were attributed to a rigid hydrogen-bonded network of hydrogen bonded nanocrystalline cellulose to form an integrated matrix as described by percolation theory.

The authors wish to acknowledge the support of the member companies of IPST at the Georgia Institute of Technology and the IPST Fellowship. Portions of this work are being used by Amit Saxena for his $\mathrm{PhD}$ requirements for graduation from the School of Chemistry and Biochemistry at the Georgia Institute of Technology, Atlanta, Georgia.

Received 24 September; accepted 4 November; published online 19 November 2010.

\section{References}

1. L. Shen, E.Worrell and M. Patel, Biofuels, Bioprod. Biorefin. 4, 25 (2010). doi:10.1002/bbb.189

2. A. Samir, F. Alloin and A. Dufresne, Biomacromolecules 6, 612 (2005).

3. A. J. Ragauskas, C. K. Williams, B. H. Davison, G. Britovsek, J. Cairney, C. A. Eckert, W. J. Frederick, J. P. Hallett, D. J. Leak, C. L. Liotta, J. R. Mielenz, R. Murphy, R. Templer and T. Tschaplinski, Science 311, 484 (2006). doi:10.1126/science.1114736

4. L. Petersson and K. Oksman, Compos. Sci. Technol. 66, 2187 (2006). doi:10.1016/j.compscitech.2005.12.010

5. J. M. Krochta, E. A. Baldwin and M. O. NisperosCarriedo, Lancaster: Technomic (1994).

6. J. Hartman, A. C. Albertsson, M. S. Lindblad and J. Sjöberg, J. Appl. Polym. Sci. 100, 2985 (2006). doi:10. 1002/app.22958

7. M. Gröndahl, L. Eriksson and P. Gatenholm, Biomacromolecules 5, 1528 (2004). doi:10.1021/ bm0499 25n

8. P. Dole, C. Joly, E. Espuche, I. Alric and N. Gontard, Carbohydr. Polym. 58, 335 (2004). doi:10.1016/j.carbpol. $\underline{2004.08 .002}$

9. B. L. Butler, P. J. Vergano, R. F. Testin, J. M. Bunn and J. L. Wiles, J. Food Sci. 61, 953 (1996). doi:10.1111/j.13652621.1996.tb10909.x
10. I. Arvanitoyannis and C. G. Biliaderis, Carbohydr. Polym. 38, 47 (1999). doi:10.1016/S0144-8617(98)00087 $\underline{-3}$

11. A. W. Rindlav, M. Stading, A. M. Hermansson and P. Gatenholm, Carbohydr. Polym. 36, 217 (1998). doi:10. $\underline{1016 / \mathrm{S} 0144-8617(98) 00025-3}$

12. P. Linder, R. Bergman, A. Bodin and P. Gatenholm, Langmuir 19, 5072 (2003). doi:10.1021/la0341355

13. K. S. Mikkonen, S. Heikkinen, A. Soovre, M. Peura, R. Serimaa, R. A. Talja, J. Appl. Polym. Sci. 114, 457 (2009). doi:10.1002/app.30513

14. U. Edlund, Y. Z. Ryberg and A. C. Albertsson, Biomacromolecules 11, 2532 (2010). doi:10.1021/bm100767g

15. M. A. S. A, Samir, F. Alloin and A. Dufresne, Biomacromolecules 6, 612 (2005).

16. Kvien, J. Sugiyama, M. Votrubec and K. Oksman, J Mater. Sci. 42, 8163 (2007). doi:10.1007/s10853-007-1699-2

17. L. Petersson, I. Kvien and K. Oksman, Composites Sci. Technol. 67, 2535 (2007). doi:10.1016/j.compscitech.2006. $\underline{12.012}$

18. X. D. Cao, H. Dong and C. M. Li, Biomacromolecules 8, 899 (2007). doi:10.1021/bm0610368

19. S. Harbaugh, N. K. Loughnane, M. Davidson, L. Narayanan, S. Trott, Y. G. Chushak and M. O. Stone, Biomacromolecules 6, 1055(2005). doi:10.1021/bm049 $\underline{291 \mathrm{k}}$

20. M. Lagaron, R. Catala and R. Gavaa, Mater. Sci. Technol. 20, 1 (2004). doi:10.1179/026708304225010442

21. M. M. De Souza Lima and R. Borsali, Macromol. Rapid. Commun. 25, 771 (2004). doi:10.1002/marc.200300268

22. M. Ioelovich, BioRes. 3, 1403 (2008).

23. S. Mikkonen, A. P. Mathew, K. Pirkkalainen, R. Serimaa, C. Xu, S. Willför, K. Oksman and M. Tenkanen, Cellulose 17, 69 (2009). doi:10.1007/s10570-009-9380-3

24. S. Mikkonen, M. I. Heikkilä, H. Helén, L. Hyvönen and M. Tenkanen, Carbohydr. Polym. 79, 1107 (2010). doi:10. $\underline{1016 / \text { j.carbpol.2009.10.049 }}$

25. P. Coughlan and G. P. Hazlewood, Hemicellulose and Hemicellulases (Eds). Portland Press Ltd, NC, U.S.A 1993.

26. A. Ebringerova and T. Heinze, Macromol. Rapid Commun. 21, 542 (2000). doi:10.1002/1521-3927(20000601)21: 9<542::AID-MARC542>3.0.CO;2-7

27. I. Gabrielli and P. Gatenholm, J. Appl. Polym. Sci. 69, 1661 (1998). doi:10.1002/(SICI)1097-4628(19980822) 69:8<1661::AID-APP19>3.0.CO;2-X 
28. A. Saxena, T. Elder, P. Shaobo and A. J. Ragauskas, Composites Part B: 40, 8 (2009).

29. A. Saxena and A. J. Ragauskas, Carbohydr. Polym. 78, 357 (2009). doi:10.1016/j.carbpol.2009.03.039

30. Y. Pu, J. Zhang, T. Elder, Y. Deng, P. Gatenholm and A. J. Ragauskas, Composites Part B: Eng. 38, 360 (2007). doi:10.1016/j.compositesb.2006.07.008

31. S. Katz, R. P. Beatson and A. M. Scallan, Sven. Papperstidn 87, 48 (1984).

32. ASTM, Standard test method for oxygen transmission rate through plastic film and sheeting using a coulometric sensor, designation D 3985-9, in: Annual Book of ASTM Standards, American Society for Testing and Materials, 1995.

33. R. T. Parry, Principles and applications of modified atmosphere packaging of foods, Blackie Academic \& Professional, England, 1 (1993).

34. Syverud and P.Stenius, Cellulose 16, 75 (2009). doi:10.1007/s10570-008-9244-2

35. A. Ebringerova and T. Heinze, Macromol. Rapid Commun. 21, 542 (2000). doi:10.1002/1521-3927(20000601)21: 9<542::AID-MARC542>3.0.CO;2-7
36. I. Gabrielli and P. Gatenholm, J. Appl. Polym. Sci. 69, 1661 (1998). doi:10.1002/(SICI)1097-4628(19980822) 69:8<1661::AID-APP19>3.0.CO;2-X

37. A. Saxena, T. Elder, P. Shaobo and A. J. Ragauskas, Composites Part B: 40, 8 (2009).

38. A. Saxena and A. J. Ragauskas, Carbohydr. Polym. 78, 357 (2009). doi:10.1016/j.carbpol.2009.03.039

39. Y. Pu, J. Zhang, T. Elder, Y. Deng, P. Gatenholm and A. J. Ragauskas, Composites Part B: Engineering 38, 360 (2007). doi:10.1016/j.compositesb.2006.07.008

40. S. Katz, R. P. Beatson and A. M. Scallan, Sven. Papperstidn 87, 48 (1984).

41. ASTM, Standard test method for oxygen transmission rate through plastic film and sheeting using a coulometric sensor, designation D 3985-9, in: Annual Book of ASTM Standards, American Society for Testing and Materials, 1995.

42. R. T. Parry, Principles and applications of modified atmosphere packaging of foods. Blackie Academic \& Professional, England, 1 (1993).

43. 34Syverud and P.Stenius, Cellulose 16, 75 (2009). doi:10.1007/s10570-008-9244-2 